\title{
LOGISTICS HUB LOCATION-SCHEDULING MODEL FOR INNER- CITY LAST MILE DELIVERIES
}

\author{
Anastasios Charisis ${ }^{1}$, Stephen Spana ${ }^{2}$, Evangelos Kaisar ${ }^{3}$, Lili Du ${ }^{4}$ \\ ${ }^{1,3}$ Freight Mobility Research Institute, Department of Civil, Environmental and Geomatics Engineering \\ Florida Atlantic University, 777 Glades Road, Boca Raton, FL, 33431, USA \\ ${ }^{2,4}$ University of Florida Transportation Institute Department of Civil and Coastal Engineering University \\ of Florida, Gainesville, FL 32611, USA
}

Received 19 December 2019; accepted 15 March 2020

\begin{abstract}
Logistics play a vital role in the prosperity of today's cities, but current urban logistics practices are proving problematic, causing negative effects such as traffic congestion and environmental impacts. This paper proposes an alternative urban logistics system, leasing hubs inside cities for designated time intervals and using handcarts for last mile deliveries. A mathematical model for selecting the locations of hubs and allocating customers, while also scheduling the optimal times during the day for leasing hubs is developed. The proposed model is compared to current delivery methods requiring door-to-door truck deliveries. It is shown that truck traveled distances decrease by more than $60 \%$. In addition, analysis shows that in certain conditions the approach can be economically competitive and successfully applied to address real problems.
\end{abstract}

Keywords: hub, location, allocation, scheduling, logistics, last-mile.

\section{Introduction}

Logistics operations have become essential to the functioning of today's economies, representing approximately $10 \%$ of the annual gross domestic product (GDP) of the U.S, and accounting for more than $37 \%$ of all jobs in the country (Harvard Business Review, 2018). Moreover, freight volumes are constantly growing and are expected to increase by nearly $29 \%$ over the next $8-10$ years (American Trucking Associations, 2018). The global use of e-commerce (e.g. Amazon, eBay, etc.) and advancing technologies implemented in logistics operations are some of the main contributors to this increase, creating high delivery expectations. Additionally, the mitigation rates to large cities are constantly increasing and it is estimated that by 2050 more than 70 percent of the global population will live in urban areas (Lee, 2014). Therefore, urban freight systems need to be able to cope with these increases as they play a major role in a city's development. An efficient and successful logistics system can improve the economic growth of an area, provide employment, attract foreign investments, and increase awareness in important issues, like environmental impacts.

A significant problem encountered in urban logistics relates to the last mile, which refers to the final step of the delivery process from a distribution center or facility to the end user and can account for up to $75 \%$ of a shipment's

${ }^{1}$ Corresponding author: acharisis2017@fau.edu 
total cost (Geavers et al., 2009). This cost is affected by (a) the high rate of unsuccessful deliveries when the delivery vehicle cannot access the urban center due to regulatory restrictions or the customer is unavailable; (b) traffic congestion and limited parking spaces in urban centers, which leads to delivery delays; and (c) underutilization of delivery vehicles' capacity (de Oliveira et al., 2014). Furthermore, an issue of major concern in today's urban transport and freight systems is increasing emission levels, which has outlined the need for sustainable development. These aspects provide a clear indication on the importance of urban freight systems to be able to provide fast, reliable, sustainable, and cost-efficient services.

The main motivation for conducting this study lies on the fact that typically freight operators locate their distribution centers outside the cities in remote areas, where the space for warehousing, transshipping, fleet deployment is larger and the land use, operations, maintenance costs lower. Most commonly, they deliver the products using trucks, traveling "door-to-door" to all the downtown destinations in urban and suburban areas. This approach is not efficient though, as it utilizes a large fleet of Heavy Goods Vehicles (HGVs) occupying urban networks for delivery operations. In addition, in order to conduct the pickups and deliveries, they are usually forced to illegally park (e.g. double park) many times during the day, a function that reduces traffic capacity and caused delays (Han et al., 2005). Moreover, many restrictions exist in urban areas regarding trucking operations during the day, making the delivery process more complex. Therefore, the aforementioned last-mile delivery method has proven to be problematic in urban areas, resulting in traffic congestion, increased emissions, delivery delays, and high last mile costs capacity (de Oliveira et al., 2014). The effects of urban freight mobility practices in cities have evolved into a crucial research domain, and many efforts are being conducted for quantifying the aforementioned problems, decreasing negative impacts, and proposing more efficient and environmentally friendly delivery methods. This is one of the pillars that the concept of sustainability in transportation systems is based on.

As can be easily concluded by the facts mentioned above, current urban logistics practices have proven unsuccessful. Therefore, it is essential that new delivery options are to be explored, which promote sustainable, environmentally friendly services which can help mitigate many of the negative effects of the ever-growing transportation networks. This study supports an alternative supply chain method for lastmile deliveries in urban congested areas. It proposes the establishment of a set small sized logistics facilities (i.e. mini-hubs) at various locations inside metropolitan areas. Since the acquisition of such properties can comprise a large capital investment and prove economically unfeasible (Muñuzuri et al., 2012) this paper proposes the leasing of the properties for designated daily time intervals required for the unloading, transshipment, and customer delivery actions. Candidate facilities include underused warehouses, lockers, or vacant parking areas-areas which could benefit from allowing different companies to lease throughout the day. Repurposing these areas for more efficient use presents a sustainable alternative to new construction. Trucks will head directly to these facilities from the main facility (distribution center that serves the whole area) and unload the cargo to the Urban Consolidation Centers (UCC) established. 
From there, the products are further delivered on foot, using handcarts to the final destinations. In addition to handcart deliveries, the proposed method, with a few adjustments, can potentially assist the bicycle logistics initiatives recently adopted by companies for the last-mile operations, as the decrease of the distances traveled for the delivery of cargo is beneficial to this aspect. Due to these facts, the study focuses on less than truckload (LTL) shipments, mostly parcel deliveries.

The planning objective of this study is the development of a novel mathematical framework for the capacitated facility location-allocation problem. This research proposes a mixed integer programming approach with constraints related to customer time deadlines and maximum distance for the allocation of nodes to hubs. The designed model selects the optimal number and locations of hubs that serve the demand with the minimum operating cost. An important aspect incorporated in the study is the leasing of the hubs for a designated daily time interval, instead of acquisition of the properties. The model, apart from selecting the locations of hubs and allocating customers to hubs, also selects the time during the day when the facilities will be leased. In each time interval costs vary, making the optimal time selection a crucial part of the formulation. This leasing function incorporated turns the problem into a joint location-allocation-scheduling one. CPLEX is used to solve the problem at hand and extract the optimal solutions, as well as to conduct additional experiments that show the decreases in Vehicle Miles Traveled (VMT) by trucks inside the cities. Last, a preliminary evaluation of the proposed method of using UCCs against the current last-mile delivery method is performed, by altering various variables of the model and identifying the cases were the hub location model is economically competitive.

The remainder of the paper is structured as follows: first, a literature review of studies related to the problem addressed in the study is conducted. Then, the problem overview, mathematical formulation and solution methodology of the model developed are described. Later, the model is applied and the results, along with the sensitivity analysis based evaluation, are presented and discussed. The study concludes with the findings of the research.

\section{Literature Review}

The literature review focuses on the two main research areas combined in the study: (i) hub location-allocation problems applied to logistics operations; (ii) urban consolidation centers analysis on their feasibility and applications.

The hub location problem is classified as a special case of the well-known location theory problems. Owen and Daskin (1998) conducted a review of studies related to facility location models, mostly focusing in the strategic nature of these problems. Yang et al. (2005) focused on finding the optimal scale and locations of city logistics terminals while minimizing the total freight transport costs. Rodriguez et al. (2007) proposed a hub location model for hub-and-spoke freight networks, considering capacity restrictions and modeling the potential congestion of arrivals at hubs as an $\mathrm{M} / \mathrm{M} / 1$ queuing system. In more recent studies, Ishfaq and Sox (2011) developed a mathematical model for the multiple-allocation $\mathrm{p}$-hub median problem for intermodal logistics networks. Muñuzuri et al. (2012) proposed a system 
of freight delivery hubs inside urban areas, solving the uncapacitated hub location problem with the goal of minimizing total transportation costs. In addition, Xifeng et al. (2013) incorporated the sustainability aspect in the logistics facility location problem, developing a method that finds the optimal trade-off between the minimization of costs, $\mathrm{CO}_{2}$ emissions and maximization of service reliability. Lin et al. (2014) proposed a heuristic for solving the intermodal terminal location problem, while introducing the terms of collaboration of unimodal road transport with intermodal transport chains. In addition, Harris et al. (2014) developed a hybrid multi-objective approach to address the capacitated facility location problem for green logistics modeling. Rao et al. (2015) also focused in the environmental impacts and created a location selection model for city logistics centers, integrating the three main aspects of sustainability (economic, environmental, and social factors). Furthermore, Lin and Lin (2016) proposed a two-stage heuristic approach to the intermodal terminal location problem, with the objective of selecting terminals that constitute an intermodal transportation network with minimal total costs. Musavi and Bozorgi-Amiri (2017) created a multiobjective model for solving the sustainable hub location-scheduling problem for perishable food supply chain with the goals of minimizing the transportation costs, maximizing product quality, and minimizing carbon emissions. Dukkanci et al. (2019) addressed the green hub location problem, finding, besides the locations of hubs and allocation of demand nodes, the speeds of the vehicles, with the goal of minimizing the total amount of emissions. Last, Charisis and Kaisar (2019) first introduced the concept of optimizing the locations of urban logistics facilities, proposing a multi- objective capacitated location-allocation model and considering constraints related to maximum node allocation range and number of customers allocated in each hub. Our current work extends this idea by incorporating the leasing aspect in the formulation and conducting a comparison with the current delivery practices.

Urban consolidation centers (UCC) provide an attractive alternative for city logistics systems. Studies the last years mostly focus on the feasibility of the concept and the conditions under which it can be successfully implemented. Van Duin et al. (2010) conducted a study to determine whether it is possible to establish UCC in the city of Hague. They concluded that the two main difficulties that lie are the allocation of costs and the unwillingness of logistics companies to cooperate, but since all parties can financially benefit from the scheme, municipalities should try to facilitate it. Roca-Riu and Estrada (2012) evaluate UCCs by proposing a cost-benefit model for carriers using an urban consolidation terminal, showing a $12-14 \%$ operational cost savings through the process. Correia et al. (2012) developed a methodology for analyzing the economic and environmental impacts of UCCs and applied it the city of Belo Horizonte. Their study showed a large reduction in emissions and number of vehicles used inside the city. In addition, Allen et al. (2012) reviewed UCC applications internationally, along with organizational, operational and financial issues required for successful implementation. They concluded by stating that UCCs have the ability to improve both supply chain performance and reduce environmental and social impacts of freight transport. Lin et al. (2016) focused on modeling urban consolidation deliveries both from logistics cost, as well as energy 
consumption perspectives. They found that under certain conditions the approach can have logistics and environmental benefits. Triantafyllou et al. (2014) identified the key issues that should be addressed during the planning phase of a UCC and demonstrated its potential transport and environmental benefits. After reviewing UK consolidation schemes, they showed that a UCC can be deemed successful when served by a group of environmentally friendly and flexible vehicles and the level of uptake is high. Gogas and Nathanail (2017) created a methodological framework for evaluating urban consolidation centers, based on selected performance indicators, such as economy, energy, mobility, policies, environment, and incorporating divergent stakeholders' interests considering conflicting business models and operations. Last, van Heeswijk et al. (2019) studied sustainable business models and administrative policies for UCCs. They performed an agent-based simulation for the city of Copenhagen, with results showing significant environmental benefits, reducing truck kilometers driven by about $65 \%$ and emissions by $70 \%$. They concluded by proposing various conditions for making UCCs viable. All studies conducted on the subject illustrate the environmental, mobility and societal prospects of establishing urban consolidation centers, while also showing that the schemes can be economically feasible.

The literature review indicates that while a large amount of work has been conducted in the optimization of logistics hub locations, its application to city logistics and Urban Consolidation Centers has not been studied. Most studies focus either in intermodal terminals or more generally in facility locations without specifying their applications. Only one study was identified dealing with the optimal locations of innercity hubs, that of Muñuzuri et al. (2012), which, as mentioned above, solved the uncapacitated $\mathrm{p}$-hub median location problem for specified curb sections where vehicles stop in order to make final deliveries. In addition, to the best knowledge of the authors, this study is the only one in the field that deals with the leasing of hubs and includes this aspect into the mathematical formulation, generating an extra scheduling dimension to the problem. Regarding the feasibility of leasing different buildings at different times of the day, distribution centers within city limits have recently been introduced to the sharing economy. Flexe is a service which provides companies with a directory of warehouses, available for flexible short-term leases. Similarly, Warehouse Exchange offers a two-sided platform in which companies and space providers are matched for distribution purposes. Clearly, space providers are eager to maximize the use of their facilities. The different leasing intervals proposed in this study would help for this to happen.

\section{Methodology}

\subsection{Problem Overview}

The problem addressed in the study is the design of a distribution hubs network inside urban areas in order to provide an alternative and innovative method of operating city logistics systems. Traditional practices involve the deployment of a large fleet 
of trucks that travel to all the downtown destinations to deliver goods. As mentioned in the introduction, a common result of this method is the illegal double parking in urban networks in order to deliver the goods. The freight parking problem has evolved into a major issue encountered in urban areas (Jaller et al., 2012). It can be easily concluded that this delivery approach has proven problematic and causes various issues in cities. Some examples are (a) noise and pollutants emissions; (b) increased fuel consumption; (c) increased logistics costs; (d) delivery delays; (e) traffic congestion; and (f) deterioration of urban infrastructure (de Oliveira et al., 2014). Additionally, certain policies have been adopted in many large metropolitan cities that relate to regulations regarding the entrance times of those types of vehicles to downtown areas. Usually these regulations restrict arrivals in peak hours, with the goal of reducing traffic impacts and pollution. It has been identified that this approach can have the opposite effect, as companies need to schedule their deliveries accordingly, and possibly use longer alternative routes to reach their destinations (Muñuzuri et al., 2012). On the contrary, this research proposes the leasing of a number of hubs inside downtown areas. Therefore, instead of traversing "door-to-door" to all the destinations, the trucks travel to these facilities and unload the products. From there they will be delivered to the final destinations (i.e. customer locations) using handcarts. The trucks used for the product deliveries will not be traveling between all the downtown destination nodes, increasing the vehicle volumes in roads and distances traveled by trucks, but will directly head to the hubs and return to their origin from predefined, low traffic routes, allowing a controllable vehicle distribution in the network. Moreover, the proposed approach explores an alternative operating method for the last mile deliveries that can potentially prove more efficient than current practices. The facilities considered act as unloading and transshipment points. Each hub has different capacity in terms of maximum cargo volumes it can handle based on the size of the facility and different fixed operating costs, depending on the size and the land use costs. Figure 1 illustrates the structure of the network developed in the study.

Apart from the practical contribution and innovation of the supply chain method proposed, the mathematical programming model developed to extract optimal solutions presents a novel approach for solving the hub location problems as well. As mentioned above, the model selects the daily time intervals when each facility should be leased. Each time interval is assumed to be of uniform length (e.g. 2, 3 hours), and the hours of operation are split into intervals according to this length (e.g. a 12 hour work day would be divided into 62 -hour intervals). Each time slot is defined by different leasing costs, as well as different average truck speeds (to account for the different traffic patterns during that time). For example, during peak hours, average speeds are slower than other times during the day, leading to alterations in the transportation costs. 


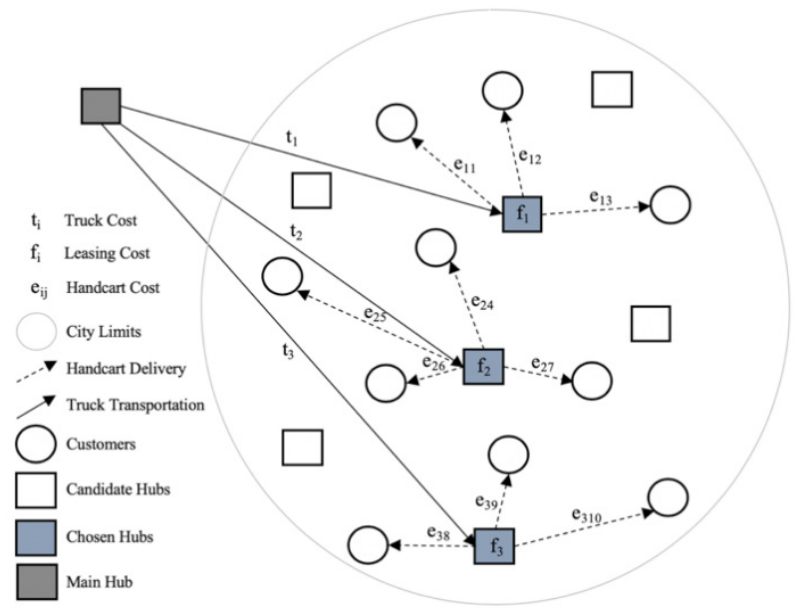

Fig. 1.

Schematic of Proposed Network

Then, the model chooses the time interval each hub should be leased, allowing different hubs to be leased at different times of the day in order to accommodate individual customer requirements.

In addition, the study adopts a time deadlines constraint, something that has scarcely been explored in the hub location problem literature. The individual time deadlines are issued by each customer as the latest acceptable time for the package to arrive. The time required to transfer the cargo from the main hub to the mini-hubs, the time for unloading and transshipping the products to the different modes, and the time to deliver the products to the final destinations is considered when determining if time deadlines are met. Finally, the proposed approach incorporates a constraint that dictates the maximum allowable distance between a node and a hub in order to allocate the node to the specific hub. Since the final deliveries are conducted using handcarts, providing as outputs locations in close proximity and easily accessible to the destinations in an important aspect of the formulation.

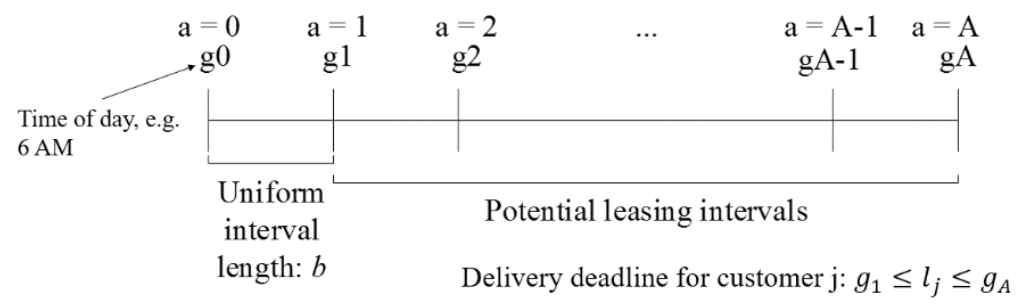

Fig. 2.

Time Interval Framework 


\subsection{Mathematical Formulation}

The model is formulated as an extension of the capacitated facility location problem, with the incorporation of the scheduling aspect of the problem. A mixed integer linear programming model (MILP) is proposed, with the goal of determining the optimal number of facilities, assignment of customers to hubs and time slots to be selected in order to satisfy all customer demand with the minimum system cost. For the formulation and development of the model, some reasonable assumptions are adopted:

- A homogeneous fleet of trucks is assumed, in terms of load capacities and fuel consumption rates. The number of trucks needed is endogenously determined using the demand at a chosen hub divided by capacity per truck (it is assumed that each company can provide the amount of trucks needed);

- It is assumed that the hub locations, usage costs and demands are fixed and known beforehand;

- The values considered for the truck and walking speeds are regarded as average speeds, incorporating acceleration, deceleration, traffic delays, etc;

- The time that it takes to unload the materials at a chosen hub is less than the length of time that the hub is leased;

- It is assumed that each vehicle departs from the main hub to the chosen facility in the time period before the chosen hub is leased in order for it to have arrived at the moment the lease starts, and also that the vehicle travels back to the main hub during the chosen interval after unloading.
The complete mathematical formulation that accurately represents the problem at hand is presented below. Let:

\section{Sets}

I Set of all candidate facilities $\mathrm{i}=1 \ldots \mathrm{I}$;

$J \quad$ Set of all destination nodes $j=1 . . . J$;

$K$ Set of trucking costs taken into consideration $\mathrm{k}=1 \ldots \mathrm{K}$;

$A \quad$ Set of time intervals $\alpha=0 \ldots \mathrm{A}$;

Note: Since we assume that all vehicles leave the main hub en route to the chosen hub during the time period previous to the time period of the lease, the time interval set index a runs from 0 to $\mathrm{A}-$ where the leasing periods are 1 ...A.

\section{Parameters}

$d$. Demand of destination node $\mathrm{j}$;

$f_{i}^{a}$ Fixed cost for using facility i during time period $\alpha$;

$t_{i}^{a}$ Transportation cost for traversing between main hub and facility i in time period $\alpha$;

$e_{i j}$ Transportation cost for traversing between hub i and customer j;

$q_{i}$ Capacity of facility i;

$c_{i j}$ Distance between facility $i$ and destination node $j$;

$h_{i}$ Distance between main hub and facility i;

$l_{j}$ Time deadline for cargo arrival in node j;

$w$ Truck capacity;

$r$ Maximum distance for allocation of facilities;

$b$ Uniform time interval length;

$v^{a}$ Average truck speed in time period $\alpha$;

$s$ Average walking speed;

$u$ Unloading time per case;

$g^{a}$ Parameter referring to time of day corresponding to index value a; 
Decision Variables

$X_{i}^{a}=\left\{\begin{array}{l}1, \text { if facility } i \text { is used in time period a } \\ 0, \text { otherwise }\end{array}\right.$

$Y_{i j}^{a}=\left\{\begin{array}{l}1, \text { if customer } j \text { is allocated to facility } i \text { at time period a } \\ 0, \text { otherwise }\end{array}\right.$

Objective Function:

Minimize $\sum_{a \in A-\{0\}} \sum_{i \in I} X_{i}^{a}\left(t_{i}^{a}+f_{i}^{a}\right)+\sum_{a \in A-\{0\}} \sum_{i \in I} \sum_{j \in J} e_{i j} Y_{i j}^{a}$

Subject to:

$\sum_{a \in A-\{0\}} \sum_{i \in I} Y_{i j}^{a}, \forall j \in J$

$Y_{i j}^{a} \leq X_{i}^{a}, \forall i \in I, j \in J, a=1 \ldots A$

$\sum_{j \in J} d_{j} Y_{i j}^{a} \leq q_{i} X_{i}^{a}, \forall i \in I, a=1 \ldots A$

$c_{i j} Y_{i j}^{a} \leq r X_{i}^{a}, \forall i \in I, j \in J, a=1 \ldots A$

$\sum_{a \in A-\{0\}} X_{i}^{a} \leq 1, \forall i \in I$

$X_{i}^{a} \frac{h_{i}}{v_{a-1}} \leq b, \forall i \in I, a=1 \ldots A$

$d_{j} u+2 c_{i j} Y_{i j}^{a} / s \leq b, \forall i \in I, j \in J, a=1 \ldots A$

$d_{j} u+\frac{h_{i}}{v_{a}} X_{i}^{a} \leq b, \forall i \in I, j \in J, a=1 \ldots A$

$g_{a} X_{i}^{a}+d_{j} u+c_{i j} Y_{i j}^{a} / s \leq l_{j} Y_{i j}^{a}, \forall i \in I, j \in J, a=1 \ldots A$

$X_{i}^{a} \in\{0,1\}, \forall i \in I, a=1 \ldots A$

$Y_{i j}^{a} \in\{0,1\}, \forall i \in I, j \in J, a=1 \ldots A$

Function (1) represents the objective of the problem, that of minimizing the total operational costs of the developed network. Total costs comprise of three components: costs for transporting the cargo to the chosen facilities; fixed costs for using each candidate hub; costs for transporting cargo from the hubs to the customers. The fixed hub costs can vary for different daily time intervals. Transportation costs depend on the number of trucks required to transfer the allocated cargo to each facility, the distances between the main facilities and the chosen hubs, the average speeds followed in the trip time interval and the truck cost per mile. The reason for incorporating the speeds in the transportation costs is to account for the different traffic patterns during the day, which will cause the trucks to operate either longer or shorter depending on the 
conditions. The number of trucks required to transport cargo to each hub is calculated by dividing the total allocated demand to a hub with the truck capacity and rounding to the highest integer. The truck cost applied in the study includes fuel costs, repair and maintenance costs, tax and insurance costs, driver payments, etc. In a similar fashion, the transportation costs for delivering cargo from the hubs to the customers depends on the distances between hubs and customers, the average walking speeds and the cost per distance for transporting on foot. Since the driver payments are incorporated in the transportation costs, the main cost incurred during the final deliveries is the truck idle time cost (Dukkanci et al., 2019).

Constraints (2)-(5) comprise the locationallocation aspects of the problem. Constraint (2) guarantees that each customer is assigned to only one facility. Inequality (3) states that a customer cannot be assigned to a facility unless it is used. Constraint (4) enforces the total demand allocated to a facility to not be greater that the facility's capacity, while inequality (5) ensures that each destination node can only be allocated to a facility if its distance to that facility is less or equal to its maximum range (radius). Next, constraints (6)-(10) illustrate the scheduling dimension of the problem for leasing the hubs. Constraint (6) ensures that each hub is chosen for at most one time slot. This represents choosing the best time to lease a single property. Constraint (7) guarantees that all vehicles can travel from the main hub to the chosen hub in the period before the building is leased (a-1). Inequality (8) ensures that the packages are delivered, processed, and the delivery people have time to deliver the packages and return to the facility during the time that the building is leased. Constraint (9) ensures that the vehicle has enough time to unload and return to the main hub during the chosen time interval a. Constraint (10) establishes the connection between the time deadline issued and the daily interval that the facility is leased for. It makes sure that the lease of the facility has initiated before the time deadline issued by the customer. In addition, it calculates the duration of all activities that occur once the building is leased, in order to compare it with the time deadline for each customer. The time deadline framework is shown graphically in Figure 2. Finally, constraints (11) and (12) are standard integrality constraints, stating that these variables are binary.

\section{Application}

\subsection{Computational Tests}

In this section, the performance of the proposed mathematical model is verified using various large size problems randomly generated. For the verification test, systemic parameters are proportionally increased, and the objective value and computation time are checked to verify the consistence of the proposed model, as well as its ability to be applied in real scenarios. Table 1 summarizes the results of the various computational experiments conducted. In all the tests 4 leasing time intervals $(\mathrm{A}=1$, ...,4) are considered. The CPLEX runs are carried out in a $2.8 \mathrm{GHz}$ processor and $8 \mathrm{~GB}$ RAM personal computer. Increase in the number of locations leads to increase in the computational time, as the model has more options to explore and the problem becomes more complex. Since the number of customers remains the same though, the total cost does not increase by a large amount, as the 
transportation costs stay in the same ranges. On the other hand, increase in the number of customers leads to a large increase in the total cost. More vehicles are required to serve the demand and more trips are conducted, translating into higher costs. The same applies for the computational times, since the problem size (hence complexity) increases.

Table 1

Computational Experiment Results

\begin{tabular}{|c|c|c|c|c|c|c|}
\hline \multirow{2}{*}{$\begin{array}{l}\text { Number of } \\
\text { Hubs }\end{array}$} & \multicolumn{2}{|c|}{20} & \multicolumn{2}{|c|}{100} & \multicolumn{2}{c|}{500} \\
\cline { 2 - 7 } & $\begin{array}{c}\text { Obj } \\
\text { Value (\$) }\end{array}$ & $\begin{array}{c}\text { CPU } \\
\text { Time (sec) }\end{array}$ & $\begin{array}{c}\text { Obj } \\
\text { Value (\$) }\end{array}$ & $\begin{array}{c}\text { CPU } \\
\text { Time (sec) }\end{array}$ & $\begin{array}{c}\text { Obj } \\
\text { Value (\$) }\end{array}$ & $\begin{array}{c}\text { CPU } \\
\text { Time (sec) }\end{array}$ \\
\hline 10 & 134 & 11.8 & 696.8 & 28.2 & 3274.96 & 171.6 \\
\hline 50 & 168.3 & 89.2 & 736.2 & 180.5 & 3607.38 & 312.6 \\
\hline 100 & 196.7 & 211.6 & 802.9 & 321.9 & 4094.79 & 581.4 \\
\hline
\end{tabular}

\subsection{Application and Output Illustration}

In this section, experiments are conducted using the proposed methodology and the results are presented in order to illustrate the function of the developed model. A small problem instance is generated, consisting of 10 candidate logistics facilities $(I=1, \ldots, 10)$, 4 leasing time intervals $(A=1, \ldots, 4)$ and 20 demand nodes $(\mathrm{J}=1, \ldots, 20)$. Truck capacities are considered 50 cases, and hub capacities to be able to approximately handle the load of 3-4 trucks. The data used for the fixed hub leasing costs in the model are based on the average rent per sq. foot paid for warehouse and distribution in the U.S. in 2018 (5.5\$/sq. $\mathrm{ft})$ and the average size of a small warehouse, brought in to a leasing time rate (Statista, 2019). The trucking costs are $1.69 \$ / \mathrm{m}$ (American Trucking Associations, 2018), while truck idle time costs are $1.38 \$$ / hour (Center for Transportation Research, 2016). The leasing time slot was considered as 4 hours, while the maximum distance for node allocation was extracted as the average of all the distances between hubs and nodes. Average trucks speeds for each interval were chosen based on weekday traffic patterns in urban areas (e.g. 7-10 a.m. speeds are lower than 10 a.m.-1 p.m. due to peak hour). Results of the optimization process and the optimal solution that serves the network with the minimum objective function value are presented in Table 2 . The table shows that 3 facilities are utilized in order to accommodate the network demand. Each node is allocated to one of those facilities, where the cargo volumes allocated to each facility do not exceed the capacity constraints. Regarding the optimal leasing times of hubs, a common pattern was observed in all the experiments conducted. The model tends to select the first indexes in order, those of earlier times in the day. This is a reasonable outcome, since in the first indexes the model has higher possibility of accommodating the deadline constraints. For example, if a deadline is at 15 hours ( 3 p.m.) and the indexes are $\mathrm{a}=1$ (7 a.m.), $\mathrm{a}=2$ (10 a.m.), a =3 (1 p.m.), if the facility use and transportation costs for these indexes are similar, the model will most likely select the first index. Last, it is worth mentioning that due to the linear and straightforward structure of the developed methodology, computational times required for the model to converge to optimal solution were less than 10 seconds. 
Table 2

Solution Output from Optimization

\begin{tabular}{|c|c|c|c|}
\hline Hubs Selected & $\begin{array}{c}\text { Time Slots } \\
\text { Selected }\end{array}$ & Customers Allocated & \\
\hline 2 & 1 & $3,4,6$, & Truck Transportation Cost \\
\hline & & $7,16,20$ & 27.6 \$ day \\
\hline 3 & 2 & $1,8,12,14$, & Hub Usage Cost \\
\hline & & $15,17,19$ & H5 \$/day \\
\hline 6 & 1 & $2,5,9,10$, & 52.4 \$ day \\
\hline & & $11,13,18$ & Total Cost \\
\hline & & & $134 \$ /$ day \\
\hline
\end{tabular}

\subsection{Evaluation and Guidelines for Implementation}

In order to test the effectiveness of the hub location model developed in the study, its performance is compared to a model that would normally be used to deliver goods in a situation where the network structure and hub establishment proposed did not exist. The model most applicable for this comparison is the Vehicle Routing Problem with Time Windows (VRPTW). This mathematical model minimizes the cost of shipment in terms of vehicles needed and distance traveled, and also ensures that deliveries are made within a certain time window (analogous to the customer time deadline parameter). Contrary to the hub location model, in which vehicles are only required to travel to the chosen hub to deliver the packages, in the VRPTW model vehicles must travel from the origin distribution center directly to each customer's location within the city limits (i.e. no buildings are chosen for leasing). We are interested in observing the performance of each model both from the freight company's perspective (total cost) and society's perspective (reduction of VMT).

The mathematical formulation used for solving the VRPTW is the one proposed by Solomon (1987). Both models are tested in CPLEX using the scenario outlined in the previous section. Since many of the parameter inputs chosen for the experiments are based on averages (or assumptions), in order for the experiments outputs to be valid and to be able to extract conclusions, we conduct multiple tests and a sensitivity analysis where we allow some parameters to vary. For each set of runs, we consider the scenario in the previous section to be the baseline scenario and then increase (or decrease) one parameter value for comparison. We observe the performance of each model both in terms of total cost and distance traveled by the trucks. This function allows us to identify the scenarios where the proposed approach results in less costs than the VRPTW.

In the first scenario, we consider the uncertainty of customer demand by allowing it to vary from $+/-30 \%$ of its original value. Results of this test are presented in Figure 3 . The results in terms of total cost show that in the baseline scenario (100\%), the VRPTW model is cheaper to implement. As demand decreases, the gap in costs between the models grows. This seems reasonable, as it would be more cost effective to deliver the goods directly under low-demand scenarios, 
rather than lease buildings (which could be operating significantly below capacity). When the demand increases, the hub location model becomes cheaper than VRPTW. This result can be explained in terms of both hub and truck capacities. The hubs - which operate below capacity in low-demand scenarios-have the flexibility of taking on more demand at no additional cost. In terms of vehicle costs, increased customer demand means that more vehicles are needed for delivery. This impacts the costs less severely in the hub location model, since the vehicles only need to travel to the hub locations (compared to VRPTW where more trips to and from the main hub are needed). These results show that the hub location model is more cost effective than the VRPTW in high customer demand scenarios.

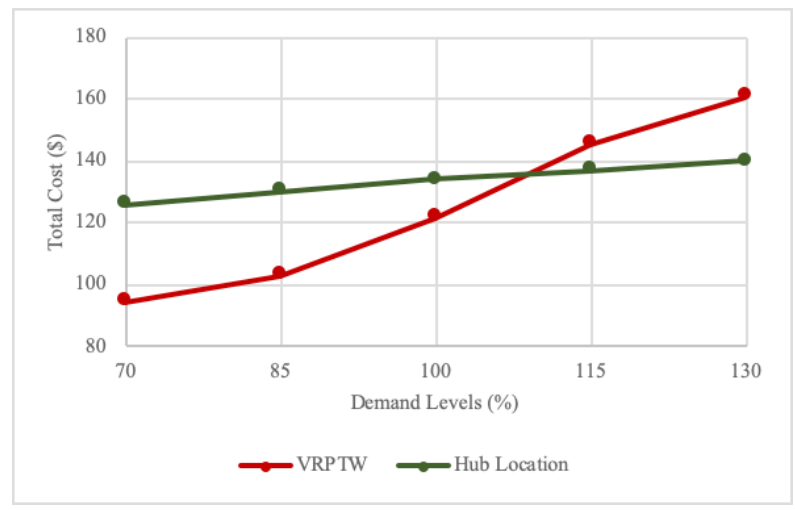

Fig. 3.

Sensitivity Analysis on Customer Demand

For the next set of runs, we vary the leasing time deadline for the chosen hubs to account for the uncertainty associated with the leasing times that are available. Outputs of this analysis are presented in Figure 4. Since no hubs are leased in the VRPTW model, the total cost for the baseline scenario is used for comparison. Here we see that for smaller time intervals, the cost is greater for the hub selection model. This makes sense, since the model would require more buildings to be leased under this scenario. With larger intervals, there is more flexibility in the hub location model to deliver within the time deadlines. It leads to the scenario where fewer buildings need to be leased, which reduces leasing costs and fixed transportation costs. This shows the value of the hub location model when longer leasing intervals are possible. 


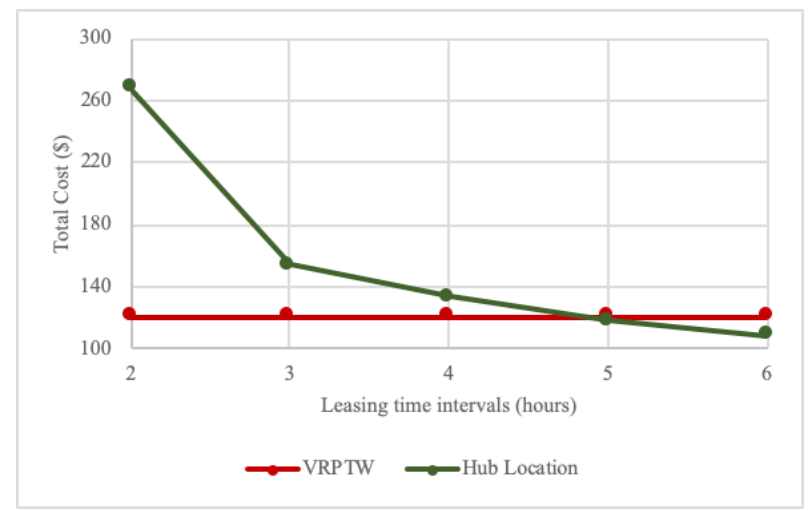

Fig. 4.

Sensitivity Analysis on Time Intervals

Finally, we allow the capacities of the chosen hubs to vary from $+/-30 \%$ of its original value to account for uncertainty in building size. Figure 5 shows the results from this experiment. Again, since no buildings are leased in the VRPTW model, the baseline scenario is used for comparison. We observe that for smaller hub capacities, the cost for the hub allocation problem is much higher than VRPTW. In these scenarios, more buildings would need to be leased to handle all of the demand (which increases leasing costs). Leasing more buildings also increases the fixed transportation cost, since more main-hub-to-chosen-hub trips are needed. As the capacities increase, we see the total cost for both models being approximately the same. This is due to the reduction in leasing costs (less need to be chosen) and fixed transportation costs (less trips needed to deliver). In general, the hub location model performs about the same as the VRPTW when building capacities are high.

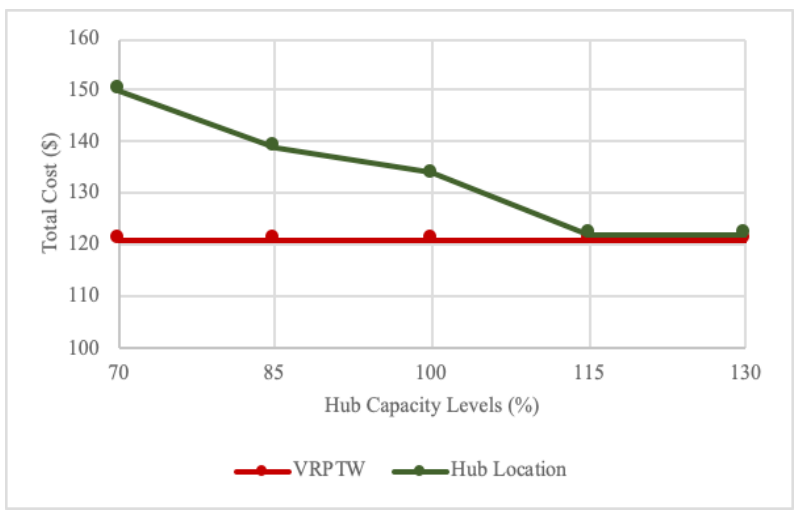

Fig. 5.

Sensitivity Analysis on Hub Capacities 
The previous results show the scenarios where it would be cost effective to choose a hub location model over the VRPTW: high demand scenarios, and scenarios where long time-leasing intervals are available. Costs are more or less the same for scenarios where high hub capacities are available. It should be reiterated that the above results are only for situations where one parameter is changed. It is reasonable to assume that different, and more favorable to the proposed approach, cost outcomes could occur when more than one parameter is altered (e.g. high demand and high hub capacity). This analysis shows that although the proposed approach seems more costly at first sight, there are cases that can result in economic competitiveness of this supply chain method.

In the sensitivity analysis, we also observed the differences in distances traveled by trucks inside cities for each model. The results were fairly consistent for each parameter that was altered, therefore we present in Figure 6 one general graph summarizing the results.
Here, 3 represents the base scenario and the lower (higher) values represent lower (higher) parameter values. It is evident from the graph that across all different scenarios, there is a dramatic reduction in truck distances traveled when the hub location model is implemented (averaging around $60 \%$ improvement). This is a very positive result from a societal perspective, because it means less vehicle miles traveled within the city limits. While this result is expected (as vehicles are not required to go door to door in the hub location model), it is still important to observe. This result illustrates the high mobility benefits of the approach proposed and could be used to favor the hub location model when the total costs in both models are approximately the same (e.g. scenarios with high hub capacities). It is also worth mentioning that these results correspond to one company implementing the method. In the case were more companies choose to adopt it, the reduction in VMTs accumulates, and the societal benefits become much greater.

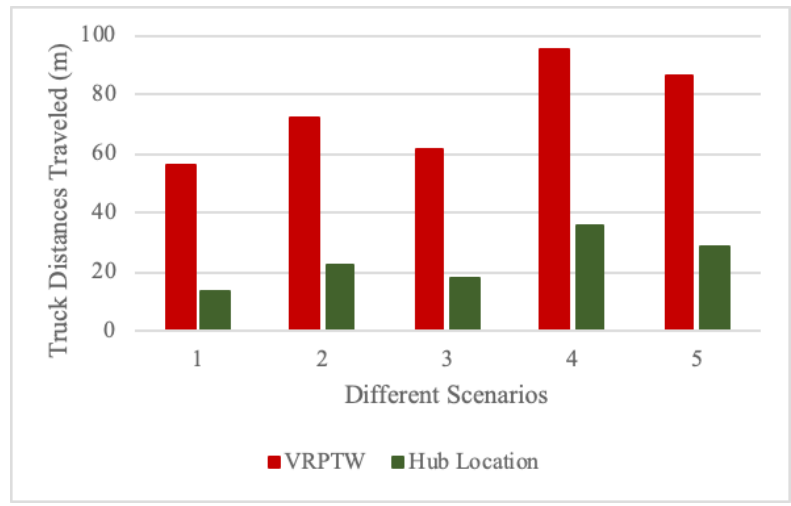

Fig. 6.

Distances Traveled Comparison 


\section{Conclusions and Extensions}

Urban logistics have become an integral component of today's cities and transportation systems. In order for them to provide sustainable and efficient services, innovative approaches are required as current practices have proven unsuccessful. This research provides an alternative method of delivering cargo to urban congested areas with the establishment of a network of low cost, small logistics facilities for handcart last-mile deliveries. It develops a novel mathematical programming model for the capacitated hub location-allocation problem with time deadlines and maximum allocation distance constraints, and solves it using CPLEX. In addition, it incorporates the aspect of leasing the hubs for different daily time intervals, a function not explored in previous studies which transforms the problem into a simultaneous locationallocation-scheduling one. Numerical experiments and a sensitivity analysis provide evidence of the function of the model and its ability to efficiently be used in real scenarios. The main advantage of the proposed methodology is its ability to capture the complexity in the process of selecting facilities and various issues encountered, such as high land use costs, limited facility capacities, delivery time requirements, restrictions related to handcart transportation, as well as traffic congestion issues. Moreover, the aspect of leasing the facilities can provide opportunities for collaborative supply chain systems, a function with high potential for both logistics companies as well as city authorities.

The flexibility of the model developed provides high potential for further extensions and even better solutions to the problem. The delivery cost component in the objective function can be easily modified to account for alternate delivery methods, including those by drone or robot. Additionally, since the problem of selecting facilities comprises of a long-term investment, the concept of uncertainty in some of the variables could be explored; for example, in the demand levels or land use costs. In addition, in order for the study to create a complete and more general network design, routing selection options could be investigated for truck and handcart operations, turning it into a simultaneous location-routing problem. This function would potentially help decrease network costs and make the proposed scheme an even more attractive option. Furthermore, collaborative schemes involving many companies making the same decision (where and when to locate distribution centers) is also a logical extension of this work.

Last, as the proposed approach provides a different supply chain method for city deliveries, evaluation of the method in real life situations is required. Preliminary evaluation results indicate that there are high prospects for successful implementation. The societal benefits incurred by applying the method and minimizing the truck trips inside cities are clear, including decreased fuel consumption, environmental impacts, traffic congestion, delivery delays and increased freight mobility. In addition, these benefits become of greater importance as more companies choose to apply the proposed approach for their lastmile deliveries, helping create sustainable urban logistics networks. Apart from the sustainability aspect, this study showed that there are scenarios where these benefits can be combined with economic competitiveness from a company perspective. Given the proper guidelines for problem aspects, such as the ones studied in the previous chapter, 
the model developed can be equally, or even less, costly than the current delivery methods applied for city deliveries. Therefore, further analysis is required to identify more variables that affect the total costs, and develop complete guidelines for successful implementation, that capture all the crucial problem aspects.

\section{Acknowledgements}

The research presented in this study is supported by the Freight Mobility Research Institute (FMRI), a Tier 1 United States Department of Transportation University Transportation Center, as part of the second year funded research projects under the number Y2R2-18.

\section{References}

Allen, J.; Browne, M.; Woodburn, A.; Leonardi, J. 2012. The role of urban consolidation centres in sustainable freight transport, Transport Reviews 32(4): 473-490.

American Trucking Associations. 2019. U.S. Freight Transportation Forecast to 2026. Available from internet: <http://www.atabusinesssolutions.com>.

Center for Transportation Research (Argonne National Laboratory). 2016. Estimation of Fuel Use by Idling Commercial Trucks. Available from internet: <http:// www.transportation.anl.gov/pdfs/T A/373.pdf $>$.

Charisis, A.; Kaisar, E. 2019. Multiobjective Capacitated Location-Allocation Model for Urban Logistics Delivery Facilities. In Proceedings of the Transportation Research Board 98 $8^{\text {th }}$ Annual Meeting, No. 19-00706.

de Assis Correia, V.; de Oliveira, L. K.; Guerra, A. L. 2012. Economical and environmental analysis of an urban consolidation center for Belo Horizonte City (Brazil), Procedia-Social and Behavioral Sciences 39: 770-782. de Oliveira, L. K.; Oliveira, B. R. P.; Correia, V. D. A. 2014. Simulation of an Urban Logistic Space for the Distribution of Goods in Belo Horizonte, Brazil, Procedia - Social and Behavioral Sciences 125(20): 496-505.

Dukkanci, O.; Peker, M.; Kara, B. Y. 2019. Green hub location problem, Transportation Research Part E: Logistics and Transportation Review 125: 116-139.

Geavers, R.; Voorde, E. V. D.; Vanelslander. T. 2009. Assessing characteristics of innovative concepts in last-mile logistics and urban distribution. In Conference proceedings of Metrans, Long Beach, EUA.

Gogas, M. A.; Nathanail, E. 2017. Evaluation of urban consolidation centers: a methodological framework, Procedia Engineering 178: 461-471.

Han, L.; Chin, S.M.; Franzese, O.; Hwang, H. 2005. Estimating the impact of pickup-and delivery-related illegal parking activities on traffic, Transportation Research Record 1906(1): 49-55.

Harris, I.; Mumford, C. L.; Naim, M. M. 2014. A Hybrid Multi-Objective Approach to Capacitated Facility Location with Flexible Store Allocation for Green Logistics Modeling, Transportation Research Part E: Logistics and Transportation Review 66: 1-22.

Harvard Business Review. 2018. The Supply Chain Economy and the Future of Good Jobs in America. Available from internet: <https://hbr.org/2018/03/ the-supply-chain-economy-and-the-future-of-goodjobs-in-america>.

Ishfaq, R.; Sox, C. R. 2011. Hub Location-Allocation in Intermodal Logistic Networks, European Journal of Operational Research 210(2): 213-230.

Jaller, M.; Holguín-Veras, J.; Hodge, S. 2013. Parking in the city: Challenges for freight traffic, Transportation Research Record 2379(1): 46-56. 
Lee, H. 2014. Urbanisation proceeding on unprecedented scale. In Proceedings of the World Cities Summit, Marina Bay Sands, Singapore.

Lin, C. C.; Chiang, Y. I.; Lin, S. W. 2014. Efficient Model and Heuristic for the Intermodal Terminal Location Problem, Computers and Operations Research 51: 41-51.

Lin, J.; Chen, Q.; Kawamura, K. 2016. Sustainability SI: logistics cost and environmental impact analyses of urban delivery consolidation strategies, Networks and Spatial Economics 16(1): 227-253.

Lin, C. C.; Lin, S. W. 2016. Two-Stage Approach to the Intermodal Terminal Location Problem, Computers and Operations Research 67: 113-119.

Muñuzuri, J.; Cortés, P.; Grosso, R.; Guadix, J.. 2012. Selecting the Location of Minihubs for Freight Delivery in Congested Downtown Areas, Journal of Computational Science 3(4): 228-237.

Musavi, M. M.; Bozorgi-Amiri, A.. 2017. A MultiObjective Sustainable Hub Location-Scheduling Problem for Perishable Food Supply Chain, Computers and Industrial Engineering, 113: 766-778.

Owen, S. H.; Daskin, M. S. 1998. Strategic Facility Location: A Review, European Journal of Operational Research 111(3): 423-447.

Rao, C.; Goh, M.; Zhao, Y.; Zheng, J. 2015. Location Selection of City Logistics Centers under Sustainability, Transportation Research Part D: Transport and Environment 36: $29-44$

Roca-Riu, M.; Estrada, M. 2012. An evaluation of urban consolidation centers through logistics systems analysis in circumstances where companies have equal market shares, Procedia-Social and Behavioral Sciences 39: 796-806.
Rodriguez, V.; Alvarez, M. J.; Barcos, L. 2007. Hub location under capacity constraints, Transportation Research Part E: Logistics and Transportation Review 43(5): 495-505.

Solomon, M.M. 1987. Algorithms for the vehicle routing and scheduling problems with time window constraints, Operations research 35(2): 254-265.

Statista. 2019. Average rent per square foot paid for industrial space in the United States in $3^{\text {rd }}$ quarter 2018, by type. Available from internet: <https://www.statista. $\mathrm{com} /$ statistics/626555/average-rent-per-square-footpaid-for-industrial-space-usa-by-type/>.

Triantafyllou, M. K.; Cherrett, T. J.; Browne, M. 2014. Urban freight consolidation centers: Case study in the UK retail sector, Transportation Research Record 2411(1): 34-44.

Van Duin, J. H. R.; Quak, H.; Muñuzuri, J. 2010. New challenges for urban consolidation centres: A case study in The Hague, Procedia-Social and Behavioral Sciences 2(3): 6177-6188.

van Heeswijk, W.; Larsen, R.; Larsen, A. 2019. An urban consolidation center in the city of Copenhagen: a simulation study, International Journal of Sustainable Transportation 13(9): 675-691.

Xifeng, T.; Ji, Z.; Peng, X. 2013. A Multi-Objective Optimization Model for Sustainable Logistics Facility Location, Transportation Research Part D: Transport and Environment 22: 45-48.

Yang, Z.; Liu, C.; Song, X. 2005. Optimizing the Scale and Spatial Location of City Logistics Terminals, Journal of the Eastern Asia Society for Transportation Studies 6: 2937-2946. 